\title{
A MODEL IN FREQUENCY DOMAIN FOR TRANSFORMATION OF FULLY DISPERSIVE NONLINEAR WAVES
}

\author{
Samira Ardani ${ }^{1}$, James M. Kaihatu ${ }^{2}$
}

\begin{abstract}
In this study, mathematical derivation and numerical verification of a wave transformation model in frequency domain is discussed. This wave model is fully dispersive and nonlinear; and is derived based on the WKB assumptions. Transforming the problem into the frequency domain and using multiple scale analysis in space and perturbation theory, the model is expanded up to second order in wave steepness. This fully dispersive nonlinear wave model is a set of evolution equations which explicitly contains quadratic near-resonant interactions. The comparison between the presented model, the existing fully dispersive model and a nearshore model with different set of laboratory and field data shows that the presented model provides significant improvements particularly at higher frequencies.
\end{abstract}

Keywords: Nonlinear nearshore wave models; Fully dispersive; Frequency domain

\section{INTRODUCTION}

Early studies of nearshore waves originate from the modeling of Boussinesq equations (Boussinesq 1872). In these equations, both weak nonlinearity and weak dispersive effects of waves are taken into account for constant depth; dispersive and nonlinearity parameters are thus kept at first order. Later, Korteweg and Vries (1895) developed a single equation for free surface elevation by combining two Boussinesq equations for a one dimensional propagation of waves in a constant depth. Mei and Mehaute (1966) and Peregrine (1967) developed Boussinesq type equations to investigate the effects of mildly varying depth. By transforming the varying-bottom Boussinesq equations in frequency domain, Freilich and Guza (1984) derived two nearshore nonlinear models (a "consistent" model and a "dispersive" model) for the shoaling region where the water depth is approximately in the range of 3 to $10 \mathrm{~m}$. They also used the result of Benney (1962) which used multiple scale expansion to formulate a series of equations which account for significant energy exchange even in only near-resonant conditions. In an attempt to improve the dispersive characteristics of Boussinesq models, Madsen et al. (1991) developed two dimensional model in time domain for constant depth by extending the velocity terms of Boussinesq equation using Taylor expansion about the bottom and added convective terms to improve the depth limitations of Boussinesq type equations. Moreover, Nwogu (1993) derived a new set of equations in time domain using the variable vertical velocity instead of a constant averaged velocity. Madsen and Sorensen (1992) extended the Boussinesq equations for mildly varying bottom slopes by developing linear shoaling properties of waves. In the frequency domain, Liu et al. (1985) applied the parabolic approximation method of Radder (1979) to modify both Boussinesq equations and KP (Kadomtsev and Petviashvili 1970) equation; The latter is a weak two-dimensional extension of the KdV equation. Chen and Liu (1995) established the frequency domain model for extended Boussinesq equations of Nwogu (1993) and developed their fourth order equation using parabolic approximation. Chen and Liu (1995) and Kaihatu and Kirby (1998) also extended the Boussinesq equations and found the optimized parameters for shoaling and dispersive terms. Later, Bredmose et al. (2004) enhanced the efficiency of the time domain Boussinesq models by applying Fast Fourier Transforms for calculation of nonlinear interaction terms.

While the Boussinesq-based nearshore models show good agreement with data, it has restrictions for application in deep water where the dispersiveness of the waves is a dominant feature. An alternative to the Boussinesq equation approach involves nonlinear extensions to linear, fully dispersive wave model. The fully dispersive nonlinear wave models have the ability to extend to intermediate and deep water. These models can under certain conditions, replicate the features of Stokes-type waves in deep water (Kaihatu 2001). Bryant (1973) developed a model for long waves based on the boundary value problem and fully dispersive features of waves and compared the model with KdV (Korteweg and Vries 1895) and Benjamin et al. (1972) equations. To establish the mathematical formulation of the model, he assumed that waves are periodic in space and nearly periodic in time and hence wave interactions are near resonant for lower frequencies. He demonstrated that fully dispersive wave models support the nonlinear triad wave-wave interaction. Moreover, Bryant (1974) demonstrated that the permanent form solution to his dispersive wave

\footnotetext{
${ }^{1}$ Department of Ocean Engineering, formerly in Zachry department of Civil Engineering, Texas A\&M University, College Station, Texas 77843-3136, United States

${ }^{2}$ Zachry Depratment of Civil Engineering, Texas A\&M University, College Station, Texas 77843-3136, United States
} 
equation recovers the third order Stokes waves amplitudes (Kaihatu 2003). However, the assumption of spatial periodicity is not useful for waves propagating over varying water depth. Mei and Unluata (1972); Keller (1988); and Boczar-Karakiewicz et al. (1986) established a system of equations for interaction of two small amplitude waves for a varying bottom based on boundary value problem. As waves propagate from deep to shallow water region, the triad wave-wave interaction terms become more predominant compare to those of quartet interaction (Agnon et al. 1993). Starting from the boundary value problem, Agnon et al. (1993) derived a one dimensional fully dispersive model that includes triad wave-wave interactions. Kaihatu and Kirby (1995) presented a two dimensional fully dispersive model using the parabolic approximation method (Radder 1979). The nonlinear part of their model consists of the triad wave-wave interactions between frequency components. Wave breaking dissipation rate term was also calculated using the approach of Mase and Kirby (1992) and included in the aforementioned model. Developing this model into two dimensions, and assuming a periodic lateral domain, Agnon and Sheremet (1997) presented a stochastic approach using bicoherence analysis to investigate the correlation between phases in nonlinear quadratic terms. In addition, Kaihatu (2001); Eldeberky and Madsen (1999); and Eldeberky (2012) improved the fully dispersive parabolic model by extending the relationship between free surface elevation and velocity potential (used in developing the frequency domain model) up to second order. According to this improvement the higher order statistics of waves can be estimated more accurately. More recent studies for generating waves evolution equations in two dimension using the boundary value problem was carried out by Janssen et al. (2006). Following Chu and Mei (1970); Liu and Dingemans (1989); Suh et al. (1990), they expanded the free surface elevation and velocity potential and studied the effects of sub and super harmonic bound waves. According to their model, both triad and quartet wave-wave interaction terms were taken into account in the model; however during wave transition process from deep to shallow water explicit shifting from one to another term is required (Tolman et al. 2013).

Although the fully dispersive nonlinear wave models do not have depth restriction such as many Boussinesq-type models, they still do not have enough performance in predicting higher frequency energy evolution (Ardani 2016). For instances, Eldeberky and Madsen (1999) does not add any improvements to the model of Kaihatu and Kirby (1995). The hybrid model of Mase and Kirby (1992) performs well, however this model is not mathematically consistent. Moreover, despite the fact that the higher frequency part of the spectra in the model of Mase and Kirby (1992) agrees well compare to other models, the performance of this model in lower frequencies is not as good as the model of Kaihatu and Kirby (1995).

Briefly, the aim of this present work is to derive a transformation model for nearshore waves which extends the model of Kaihatu and Kirby (1995) to improve the performance of the model in higher frequencies. This study consists of developing a model that connects the deep water physics with those of shallow water. This model is expected to be mathematically consistent, which means that the transformation of energy from deep to shallow water and transitions between these asymptotes in intermediate water depth matches properly. We will show that the model would be better able to estimate spectral density at high frequencies. In section 2, we introduce the mathematical formulation of the proposed model in frequency domain. The derivation is based on the boundary value problem for velocity potential, $\Phi$; and the boundary condition equations are extended up to the third order of nonlinearity $\left(\mathrm{O}\left(\epsilon^{3}\right)\right)$ where $\epsilon=k a$, in which $a$ is the amplitude and $k$ is the wave number. In section 3, we discuss the results; and evaluate the numerical model using a wide range of laboratory and field datasets. We finally present the conclusion in section 4 .

\section{MATHEMATICAL DERIVATION OF THE MODEL IN FREQUENCY DOMAIN}

Most of the previous nearshore models have some limitations for higher frequency bands and they are not able to estimate higher order statistical parameters of waves accurately. To improve the performance of nearshore models, we derive a model based on the boundary value problem and WKB assumptions. Assuming the fluid is inviscid and irrotational, the boundary value problem for velocity potential in nondimensional form is formulated as

$$
\begin{array}{cc}
\nabla_{h}^{2} \phi+\phi_{z z}=0 & -h<z<\epsilon \eta \\
\phi_{z}=-\nabla_{h} h \cdot \nabla_{h} \phi & z=-h \\
\eta+\phi_{t}+\frac{\epsilon}{2}\left[\left(\nabla \phi_{h}\right)^{2}+\phi_{z}^{2}\right]=0 & z=\epsilon \eta \\
\eta_{t}-\phi_{z}+\epsilon \nabla_{h} \eta \cdot \nabla_{h} \phi=0 & z=\epsilon \eta
\end{array}
$$


Where $\nabla=(\partial / \partial x, \partial / \partial y)$ and subscripts of time denote partial derivatives. $\phi$ and $\eta$ are velocity potential and free surface elevation respectively, $h$ is the water depth and $\epsilon$ ( $k a$, where a is the characteristic amplitude) is the wave steepness or nonlinearity parameter. We expand the last two equations above, dynamic and kinematic free surface boundary conditions respectively, using Taylor series about $z=0$

$$
\begin{gathered}
\eta+\phi_{t}+\epsilon \eta \phi_{z t}+\frac{\epsilon^{2}}{2} \eta^{2} \phi_{z z t}+\frac{\epsilon}{2}\left[\left(\nabla_{h} \phi\right)^{2}+\phi_{z}^{2}\right]+\frac{\epsilon^{2}}{2} \eta\left[\left(\nabla_{h} \phi\right)^{2}+\phi_{z}^{2}\right]_{z}+H O T \\
\eta_{t}-\phi_{z}+\epsilon \nabla_{h} \eta \cdot \nabla_{h} \phi-\epsilon \eta \phi_{z z}-\frac{\epsilon^{2}}{2} \eta^{2} \phi_{z z z}+\frac{\epsilon^{2}}{2} \eta\left[\nabla_{h} \eta \cdot \nabla_{h} \phi\right]+H O T
\end{gathered}
$$

After combining the two free surface boundary conditions together, perturbation analysis, substituting $\eta=-\phi_{t}$ and Laplace governing equation and dimensionalizing, the wave equation for each order is written as,

$$
\phi_{z}=-\frac{1}{g}\left[\phi_{t t}+-\frac{1}{2}\left(\nabla_{h} \phi\right)_{t}^{2}+-\frac{1}{2}\left(\phi_{z}\right)_{t}^{2}-\frac{1}{2 g}\left(\phi_{t}\right)_{z t}^{2}+\nabla_{h} \cdot\left(\phi_{t} \nabla_{h} \phi\right)\right] \quad z=0
$$

Following the method of Smith and Sprinks (1975), the linear mild slope equation is constructed by separation of the depth dependency term and summation of the solutions

$$
\phi=\sum_{n=1}^{\infty} f_{n}\left(k_{n}, h, z\right) \widetilde{\Phi}_{n}\left(x, y, k_{n}, \omega_{n}, t\right)
$$

and

$$
f_{n}=\frac{\cosh k_{n}(h+z)}{\cosh k_{n} h}
$$

where $f_{n}$ is the depth dependency term, $k_{n}$ in the total wave number and $\omega_{n}$ is the angular frequency. Moreover, Kaihatu and Kirby (1995) applied Green's second identity to $f_{n}$ and $\widetilde{\Phi}_{n}$ as follows

$$
\int_{-h}^{0}\left\{f_{n} \widetilde{\Phi}_{n}-\widetilde{\Phi}_{n} f_{n z z}\right\} d z=\left[f_{n} \widetilde{\Phi}_{n z}-\widetilde{\Phi}_{n} f_{n z}\right]_{-h}^{0}
$$

Plugging $f_{n}(0)=1$ and Substituting the boundary condition equation (equation (7)) into (10), the primary form of the equation is written as

second order:

$$
\frac{1}{g} \widetilde{\Phi}_{n_{t t}}+F_{1} \widetilde{\Phi}_{n}-\nabla \cdot\left[F_{2} \nabla \widetilde{\Phi}_{n}\right]=-\frac{1}{g}\left[\frac{1}{2}\left(\nabla_{h} \widetilde{\Phi}_{n}\right)_{t}^{2}+\frac{1}{2}\left(\widetilde{\Phi}_{n z}\right)_{t}^{2}-\frac{1}{2 g}\left(\widetilde{\Phi}_{n t}\right)_{z t}^{2}+\nabla_{h} \cdot\left(\widetilde{\Phi}_{n t} \nabla_{h} \widetilde{\Phi}_{n}\right)\right]
$$

where

$$
\begin{aligned}
& F_{1}=\frac{1}{\cosh ^{2} k_{n} h}\left[\frac{\cosh 2 k_{n} h}{4 k_{n}}-\frac{1}{4 k_{n}}-\frac{h}{2}\right] \\
& F_{2}=\frac{1}{\cosh ^{2} k_{n} h}\left[\frac{\cosh 2 k_{n} h}{4 k_{n}}-\frac{1}{4 k_{n}}+\frac{h}{2}\right]
\end{aligned}
$$

The nonlinearity in the model occurs due to the existence of velocity potential products in the boundary condition equations and consequently, yields the products of amplitudes. The nonlinear terms at right-hand side of (11), are treated as triad wave-wave interaction to exchange energy among frequencies. Herein, two arbitrary frequency modes, $l$ and $m$ are chosen 


$$
\begin{aligned}
\frac{1}{g} \widetilde{\Phi}_{n_{t t}}+F_{1} \widetilde{\Phi}_{n}-\nabla \cdot\left[F_{2} \nabla \widetilde{\Phi}_{n}\right]= & \\
& \frac{1}{2}\left[\sum _ { l } \sum _ { m } \left\{\frac{k_{l} \tanh k_{l} h+k_{m} \tanh k_{m} h}{g}\left(\widetilde{\Phi}_{l_{t}} \widetilde{\Phi}_{m_{t}}\right)_{t}\right.\right. \\
& \left.-\left(k_{l} \tanh k_{l} h\right)\left(k_{m} \tanh k_{m} h\right)\left(\widetilde{\Phi}_{l} \widetilde{\Phi}_{m}\right)_{t}\right\} \\
& -\sum_{l} \sum_{m}\left\{\left[\nabla_{n} \widetilde{\Phi}_{l} \cdot \nabla_{n} \widetilde{\Phi}_{m}\right]_{t}+\nabla_{n} \cdot\left[\widetilde{\Phi}_{l_{t}} \nabla_{n} \widetilde{\Phi}_{m}\right]\right. \\
& \left.\left.+\nabla n \cdot\left[\widetilde{\Phi}_{m_{t}} \nabla_{n} \widetilde{\Phi}_{l}\right]\right\}\right]
\end{aligned}
$$

In order to split up the spatial quantities (quantities that are dependent on $x$ ), the multiple scale analysis is used. One of the advantages of multiple scale analysis is that the quantity is physically separated at each order. It is assumed that the spatial variable is defined for different scales

$$
\begin{aligned}
& x=x+\epsilon x \\
& x=x+X_{1}
\end{aligned}
$$

Therefore, for each order, there are some other terms in the equations obtained by slow varying assumption of $x$ that has not been shown here. According to Freilich and Guza (1984), the amplitude and phase function is assumed to vary slowly in $\mathrm{x}$. Hence the solutions for each order is defined in the following form

$$
\widetilde{\Phi}_{1 n}=\frac{-i g}{2 \omega_{n}} a_{n}\left(X_{1}\right) e^{i \Psi_{n}}
$$

where $\Psi_{n}=\psi_{n}(x)-\omega_{n} t$. This is referred to as a "phase function" and includes both spatial and temporal terms. The real-valued amplitude is $a_{n}$.

The time periodicity will be canceled out by taking Fourier series in time

$$
\widetilde{\Phi}_{n}=\frac{\widetilde{\phi}_{n}}{2} e^{-i \omega_{n} t}+\frac{\widetilde{\phi}_{n}^{*}}{2} e^{i \omega_{n} t}
$$

After eliminating the time dependency, the second order triads, (equation (14)), is written as

$$
\begin{aligned}
\frac{1}{g} \omega^{2} \widetilde{\phi}_{n}-F_{1} \widetilde{\phi}_{n}+\nabla \cdot\left[F_{2} \nabla \widetilde{\phi}_{n}\right]= & \\
& \frac{-i}{4 g}\left[\sum _ { l } ^ { n - 1 } \left\{2 \omega_{n} \nabla_{n} \widetilde{\phi}_{l} \cdot \nabla_{n} \widetilde{\phi}_{n-l}+\omega_{l} \widetilde{\phi}_{l} \nabla_{n}^{2} \widetilde{\phi}_{n-l}+\omega_{n-l} \widetilde{\phi}_{n-l} \nabla^{2} \widetilde{\phi}_{l}\right.\right. \\
& +\left[\frac{k_{l} \tanh k_{l} h+k_{n-l} \tanh k_{n-l} h}{g}\left(\omega_{l}^{2} \omega_{n-l}+\omega_{l} \omega_{n-l}^{2}\right)\right. \\
& \left.\left.\left.\left.+\omega_{n} \cdot\left(k_{l} \tanh k_{l} h\right)\left(k_{n-l} \tanh k_{n-l}\right)\right] \widetilde{\phi}_{l} \widetilde{\phi}_{n-l}\right)\right\}\right]_{n} \\
& -\frac{i}{2 g}\left[\sum _ { l } ^ { N - l } \left\{2 \omega_{n} \nabla_{n} \widetilde{\phi}_{l}^{*} \cdot \nabla_{n} \widetilde{\phi}_{n+l}+\omega_{n+l} \nabla_{n}^{2} \widetilde{\phi}_{l}^{*}-\omega_{l} \widetilde{\phi}_{l}^{*} \nabla^{2} \widetilde{\phi}_{n+l}\right.\right. \\
& +\left[\frac{k_{l} \tanh k_{l} h+k_{n+l} \tanh k_{n+l} h}{g}\left(\omega_{l}^{2} \omega_{n+l}-\omega_{l} \omega_{n+l}^{2}\right)\right. \\
& \left.\left.\left.+\omega_{n} \cdot\left(k_{l} \tanh k_{l} h\right)\left(k_{n+l} \tanh k_{n+l}\right)\right] \widetilde{\phi}_{l}^{*} \widetilde{\phi}_{n+l}\right)\right]
\end{aligned}
$$

The velocity potential for second and third orders is further defined as 
Trial A
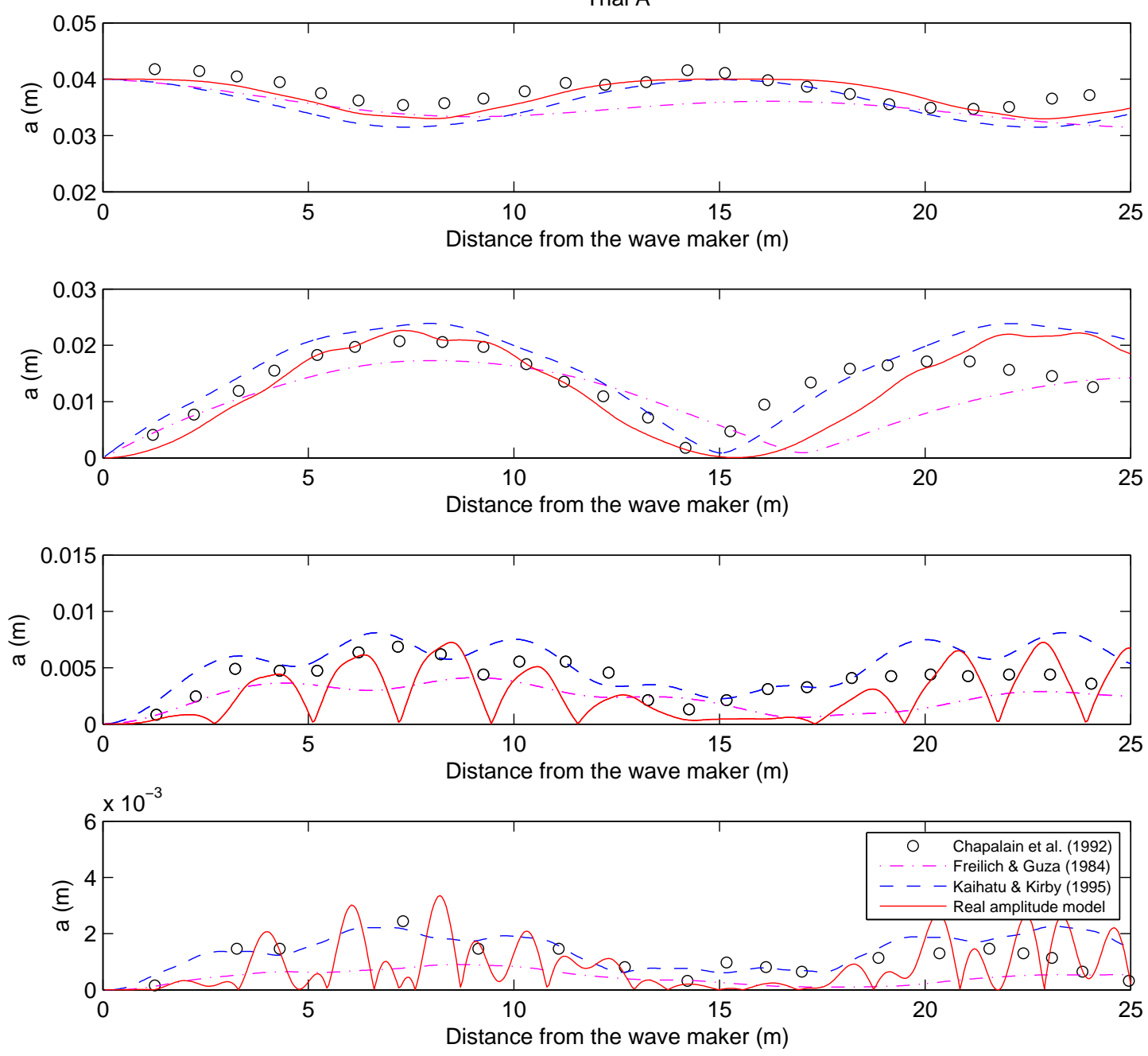

Figure 1: Comparison of Chapalain et al. (1992) experiment with real amplitude model, Kaihatu and Kirby (1995) mild slope model and the consistent model of Freilich and Guza (1984), $h=0.4 \mathrm{~m}$ and $T=2.5 \mathrm{~S}$

$$
\widetilde{\phi}_{1 n}=\frac{-i g}{\omega_{n}} a\left(X_{1}\right) e^{i \psi}
$$

where $\psi=\psi_{n}(x)=\int k_{n} d x$. Following the method of Freilich and Guza 1984, the first and second spatial derivatives of $\psi$ in second order is written as,

$$
\begin{aligned}
& \frac{d \psi_{n}}{d x}=\bar{k}+\epsilon k_{1} \\
& \frac{d^{2} \psi_{n}}{d x^{2}}=\epsilon \frac{d \bar{k}}{d X_{1}}
\end{aligned}
$$

where $\bar{k}$ is the linear or reference wave number and it is defined using the linear dispersion relation

$$
\omega_{n}^{2}=g \bar{k} \tanh \bar{k} h
$$

In (21), $k_{1}$ is the modified wave number due to bottom slope.

By substituting (20), (21) and (22) into (19) and combining linear and nonlinear terms 


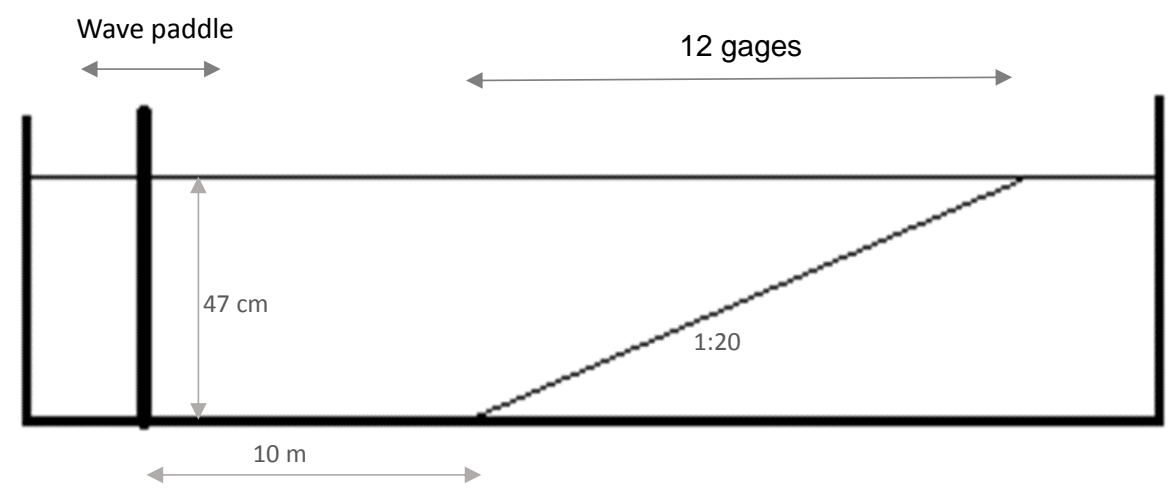

Figure 2: Mase and Kirby (1992) experimental setup

$$
\begin{aligned}
2 F_{2} a \bar{k} k_{1}+2 i F_{2} \bar{k} \frac{d a}{d x}+i F_{2} \frac{d \bar{k}}{d x} a+i F_{2 x} a \bar{k}= & \sum_{i=1}^{n-1} R a_{l} a_{n-l} e^{i\left(\psi_{l}+\psi_{n-l}-\psi_{n}\right)} \\
& +\sum_{i=1}^{N-n} 2 S a_{l} a_{n+l} e^{i\left(\psi_{n+l}-\psi_{l}-\psi_{n}\right)}
\end{aligned}
$$

where

$$
\begin{array}{r}
R=\quad \frac{\omega_{n}}{\omega_{l} \cdot \omega_{n-1}}\left[2 \omega_{n} k_{l} k_{n-l}+\omega_{l} k_{n-l}^{2}+\omega_{n-l} k_{l}^{2}-\left(\frac{k_{l} \tanh k_{l} h+k_{n-l} \tanh k_{n-l} h}{g}\right) .\right. \\
\left.\left(\omega_{l}^{2} \omega_{n-l}+\omega_{l} \omega_{n-l}^{2}\right)-\omega_{n}\left(k_{l} \tanh k_{l} h\right) \cdot\left(k_{n-l} \tanh k_{n-l} h\right)\right] \\
S=\quad \frac{\omega_{n}}{\omega_{l} \cdot \omega_{n+1}}\left[2 \omega_{n} k_{l} k_{n+l}+\omega_{l} k_{n+l}^{2}-\omega_{n+l} k_{l}^{2}+\left(\frac{k_{l} \tanh k_{l} h+k_{n+l} \tanh k_{n+l} h}{g}\right) .\right. \\
\left.\left(\omega_{l}^{2} \omega_{n+l}-\omega_{l} \omega_{n+l}^{2}\right)+\omega_{n}\left(k_{l} \tanh k_{l} h\right) .\left(k_{n+l} \tanh k_{n+l} h\right)\right]
\end{array}
$$

$\frac{d \bar{k}}{d x}$ is calculated analytically by differentiating the linear dispersion relation

$$
\frac{d \bar{k}}{d x}=\frac{d h}{d x}\left(\frac{-\bar{k}^{2} \sinh ^{-2} \bar{k} h}{\tanh \bar{k} h+\bar{k} h}\right)
$$

where $\frac{d h}{d x}$ is the variation of bathymetry and it is calculated for each step using the first order finite difference schemes.

Since $a_{n}$ in the above equation is real valued, the equation is divided into real and imaginary part and finally, the resulting system of equations are solved numerically. The homogeneous part of this second order solution is cancelled out since it has been already considered once in the first order solution. The real part of the equation (24) is used to calculate $k_{1}$ and the imaginary part is solved for $a$

$$
k_{1}=\frac{1 .}{2 F_{2} a \bar{k}}\left[\sum_{i=1}^{n-1} R a_{l} a_{n-l} \cos \left(\psi_{l}+\psi_{n-l}-\psi_{n}\right)\right.
$$

$$
\left.+\sum_{i=1}^{N-n} 2 S a_{l} a_{n+l} \quad \cos \left(\psi_{n+l}-\psi_{l}-\psi_{n}\right)\right]
$$



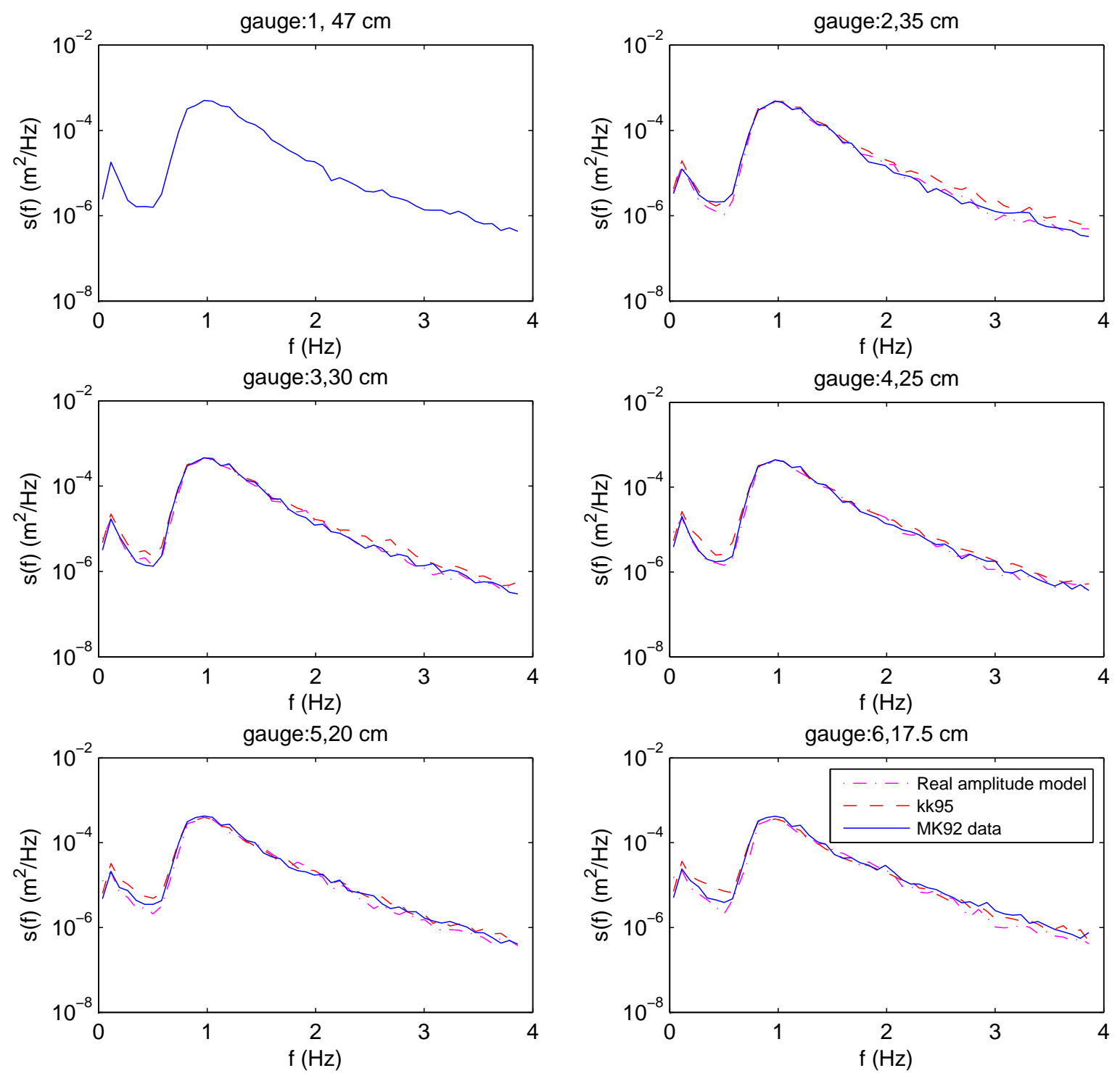

Figure 3: Comparison of Mase and Kirby (1992) experiments (gauges 1-6) with real amplitude model and the mild slope model of Kaihatu and Kirby (1995)

and

$$
\begin{aligned}
& \frac{d a}{d x}+\left(\frac{1}{2 \bar{k}} \frac{d \bar{k}}{d x}+\frac{F_{2 x}}{2 F_{2}}\right) a= \\
& \frac{-i}{2 F_{2} \bar{k}}\left[\sum_{i=1}^{n-1} R a_{l} a_{n-l} \sin \left(\psi_{l}+\psi_{n-l}-\psi_{n}\right)\right. \\
& \left.+\sum_{i=1}^{N-n} 2 S a_{l} a_{n+l} \sin \left(\psi_{n+l}-\psi_{l}-\psi_{n}\right)\right]
\end{aligned}
$$

One problem with incorporation of energy dissipation mechanism based on wave statistics (e.g. Thornton and Guza 1983) is that some ad hoc means of specifying the frequency dependence of the mechanism is required. This is because these models usually assume that the wave field is narrow-banded, in which a single average frequency is considered sufficiently descriptive. Mase and Kirby (1992) provided arguments for using a frequency-squared dependence, while Eldeberky and Battjes (1996) assume that the dissipation 

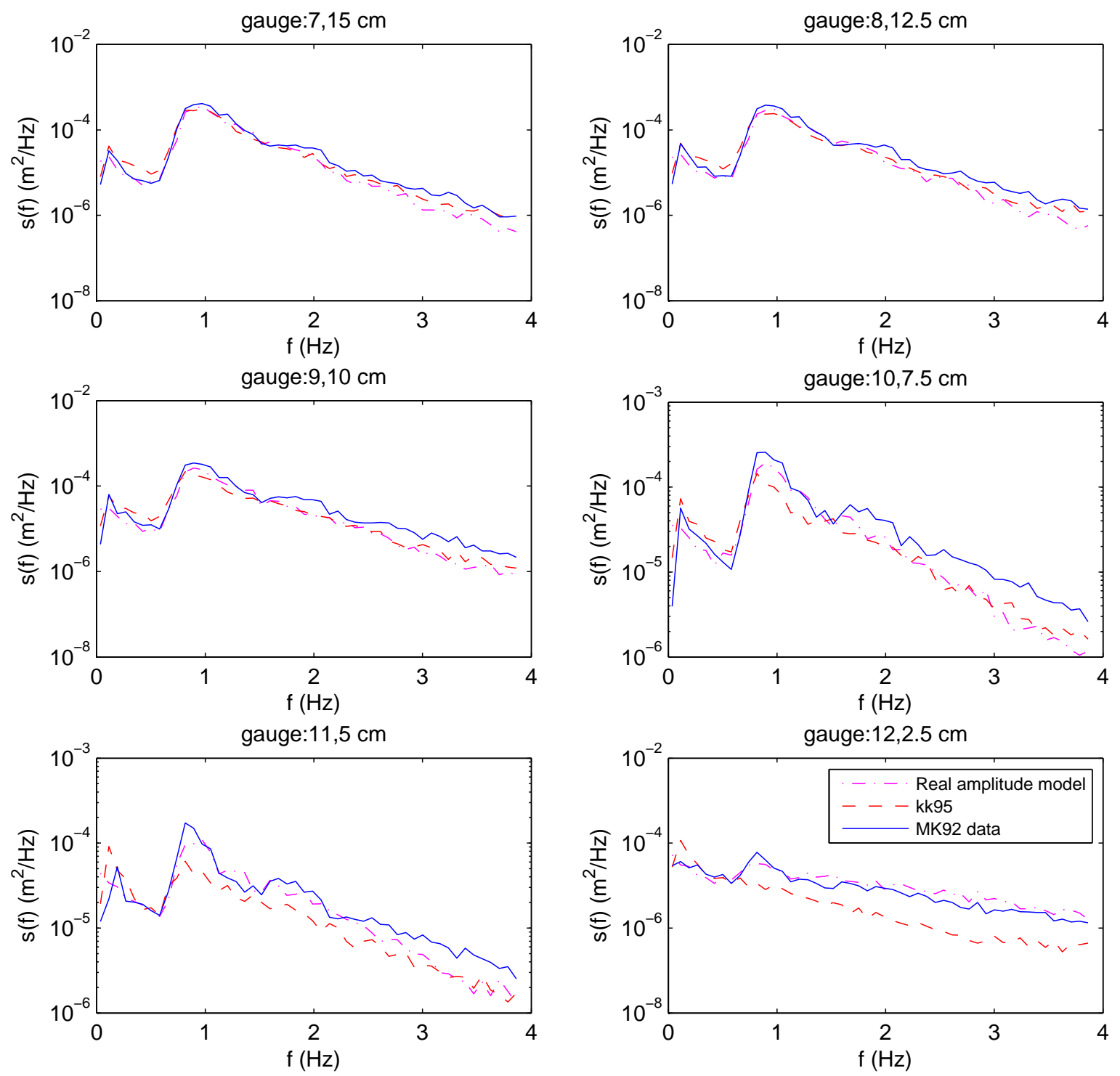

Figure 4: Comparison of Mase and Kirby (1992) experiments (gauges 7-12) with real amplitude model and the mild slope model of Kaihatu and Kirby (1995)

is constant over frequency. Both provide evidence for the frequency-squared distribution, but Kirby and Kaihatu (1996) note that the inability to easily model wave motions up to the Nyquist frequency might affect how well this particular distribution would work.

Following Kirby et al. (1992), Mase and Kirby (1992) and Kaihatu and Kirby (1995), a dissipation term, $\alpha_{n} a_{n}$, is added to the linear part of the evolution equation (equation (29)) to have a better estimation of waves in the surf zone. The dissipation term $\alpha_{n}$ is

$$
\begin{gathered}
\alpha_{n}=\alpha_{n 0}+\left(\frac{f_{n}}{f_{\text {peak }}}\right)^{2} \alpha_{n 1} \\
\alpha_{n 0}=F \beta(x) \\
\alpha_{n 1}=\left(\beta(x)-\alpha_{n 0}\right) \frac{f_{\text {peak }}^{2} \sum_{n=1}^{N}\left|A_{n}\right|^{2}}{\sum_{n=1}^{N} f_{n}^{2}\left|A_{n}\right|^{2}}
\end{gathered}
$$




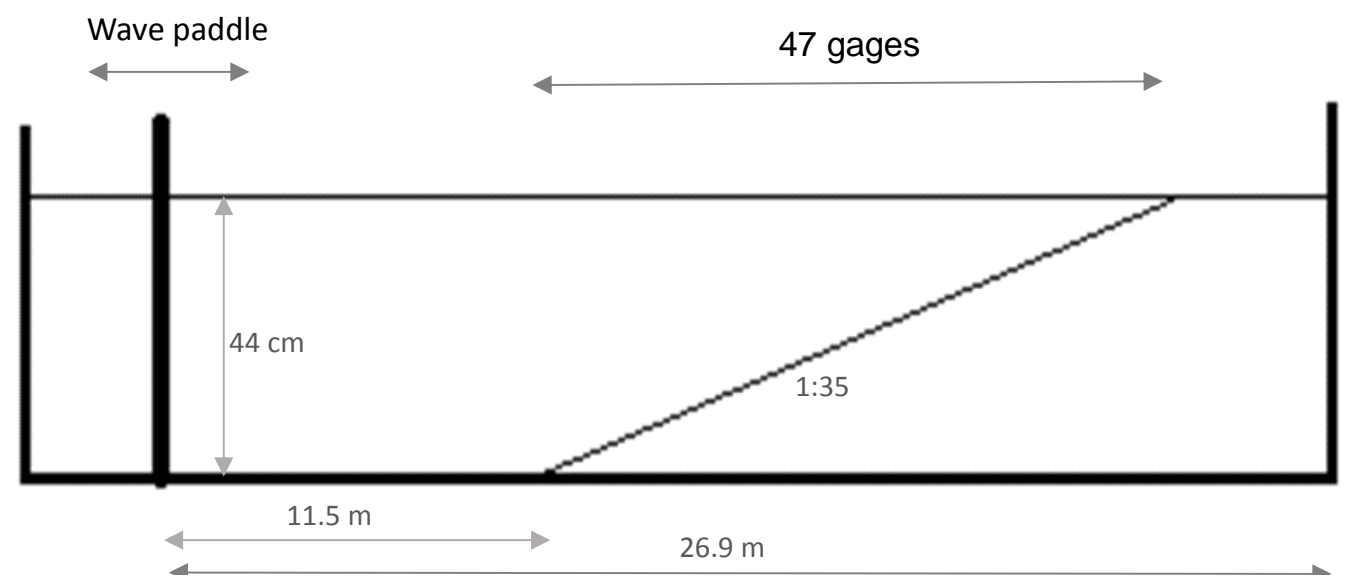

Figure 5: Bowen and Kirby (1994) experimental setup

$$
\begin{gathered}
\beta(x)=\frac{3 \sqrt{\pi}}{4 \sqrt{g h}} \frac{B^{3} f_{\text {peak }} H_{r m s}^{5}}{\gamma^{4} h^{5}} \\
H_{r m s}=2 \sqrt{\sum_{n=1}^{N}\left|A_{n}\right|^{2}}
\end{gathered}
$$

where $\beta$ is the energy dissipation rate as dictated by Thornton and Guza (1983), $f_{\text {peak }}$ is the peak frequency, $f$ is the frequency component and $B, \gamma$ and $F$ are free parameters; $F$ denotes the frequency dependency of the dissipation term. According to Kaihatu and Kirby (1996), $F=1.0$ determines that there is no dependence on frequency for dissipation, while $F=0$ leads to an $f_{n}^{2}$ dependency for dissipation. Later, Kaihatu et al. (2018) indicated that the value of $F$ may be affected by truncating the modeled frequency components below the Nyquist limit. In this study, the frequency dependency parameter for all laboratory and field tests was chosen to be $F=0$ to have the full dependency of dissipation to frequency.

\section{NUMERICAL ANALYSIS AND RESULTS}

Verification of the model using Harmonic test of Chapalain et al. (1992)

Early experiment on nonlinear wave-wave interactions was carried out by Boczar-Karakiewicz et al. (1987) for exchanging energy between the first and second harmonics. Chapalain et al. (1992) investigated the energy exchange between first four harmonics for weakly nonlinear and dispersive long waves for a constant depth. This experimental model is simple but useful way to make sure about the performance of the numerical model. To verify the ability of the model in terms of transferring the energy into higher harmonics, the experimental tests of the Chapalain et al. (1992) was compared with the numerical wave model. In order to replicate their model, the bottom variation is turned off from the model and the first four harmonics has chosen for the comparison. Figure 1 shows the comparison of three models; model of Kaihatu and Kirby (1995), consistent model of Freilich and Guza (1984) and the real amplitude model with test A of this experiment. It is apparent that the model of Freilich and Guza (1984) underpredicts the free surface amplitudes for all test cases and harmonics. The model of Kaihatu and Kirby (1995) shows better agreement with the data compare to the other two models. This discrepancy between the model of Freilich and Guza (1984) and the experimental data probably is due to the violation of shallow water limit that governs this model. The pursuit model shows a high degree of oscillation at the third and fourth harmonics, which might suggest that splitting of the wave number into components with different scales of variability might not be appropriate for this case and more investigation is required.

\section{Comparison of the model with laboratory datasets for random waves}

For comparison to experiment, the time series at the initializing gage is required. By running the model, the complex Fourier components is obtained for each gage location and each realization. The power spectra is calculated using the real and imaginary parts of the Fourier components. Bartlett averaging is used to 

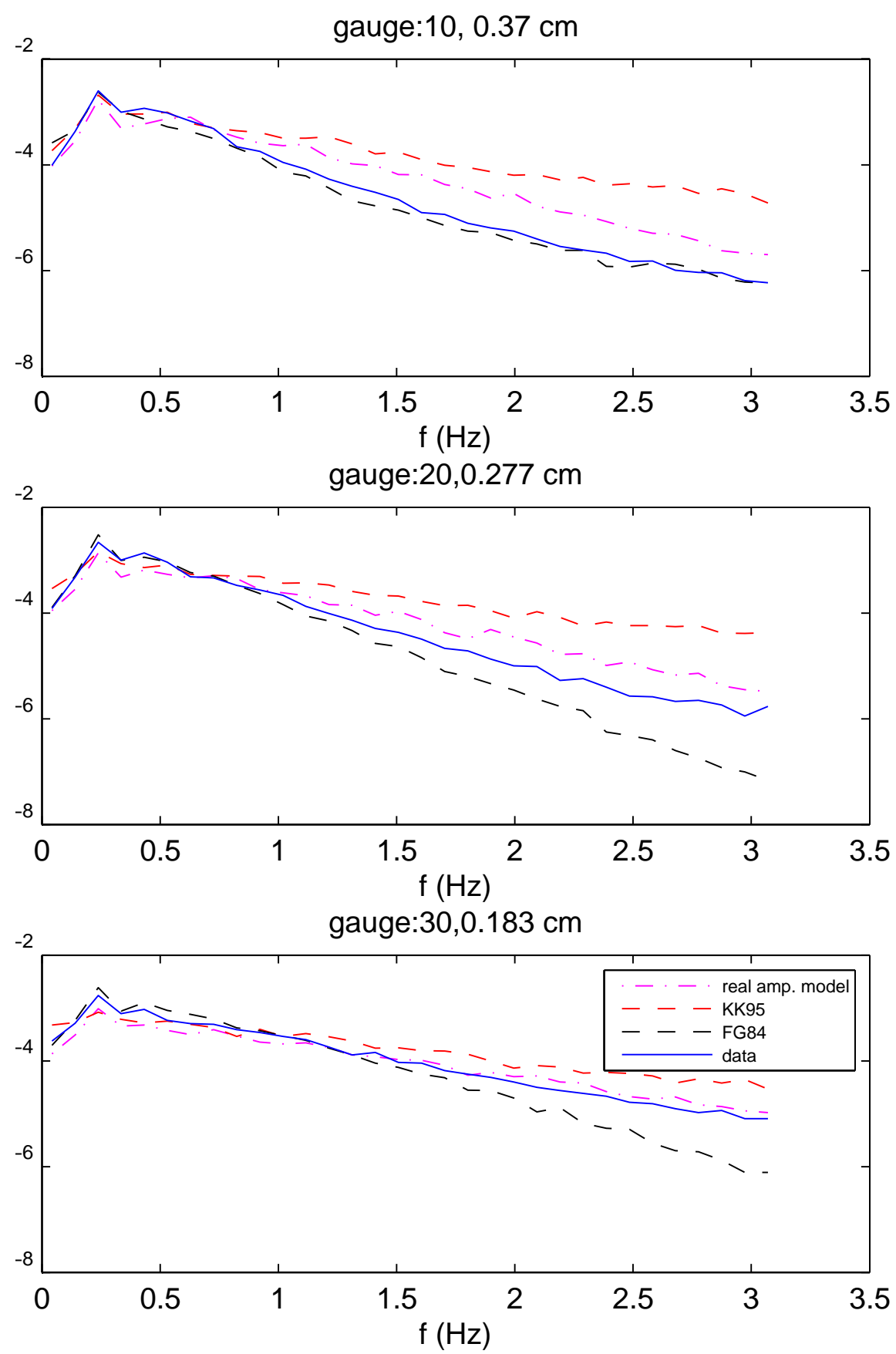

Figure 6: Comparison of Bowen and Kirby (1994) experiments with real amplitude model, Kaihatu and Kirby (1995) mild slope model and the consistent model of Freilich and Guza (1984) 

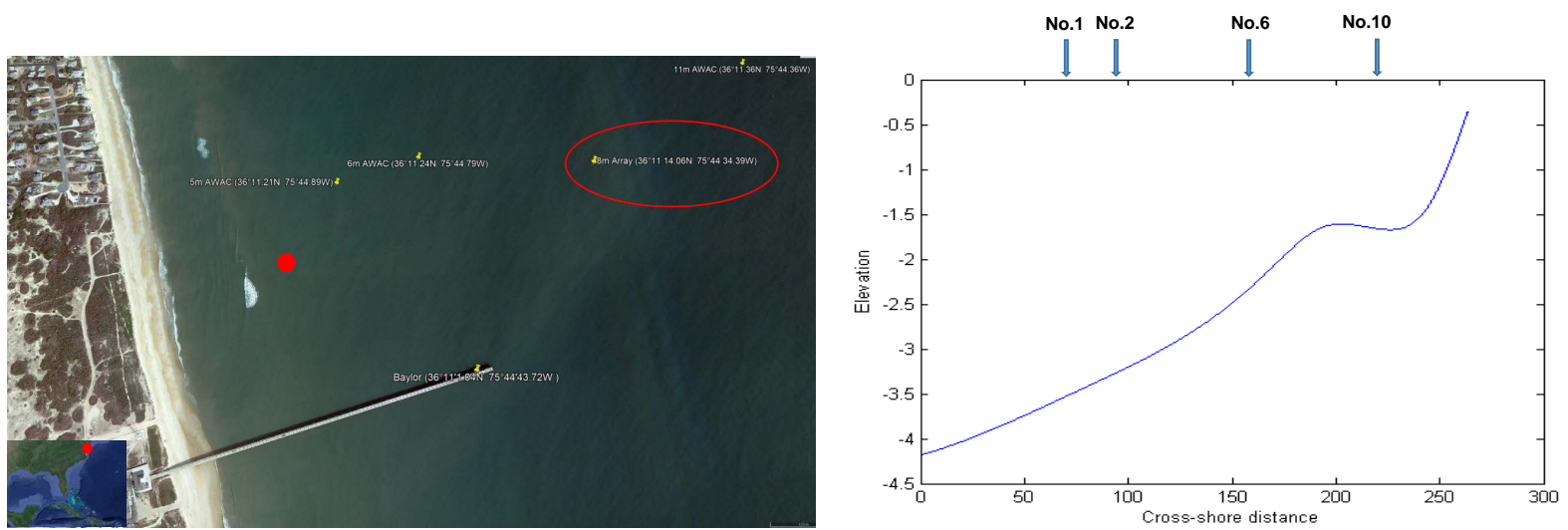

Figure 7: Schematic view of Duck 94 experiment, U.S. Army Field Research Facility at Duck, North Carolina, USA (Birkemeier and Thornton 1994), Left: Plan view; Right: Section view

take the average of the calculated power for all realizations. Band averaging is used further to reduce the noise in the power spectrum and smooth the spectral estimate.

To test the model performance, we use two laboratory experimental data sets. The results from Mase and Kirby (1992), who conducted a set of experiments in two cases for investigation of shoaling and breaking characteristics of random waves. Case 2 of this experiment is a most suitable one for testing dispersive models because the relative depth is high enough $(k h=1.9)$ and is outside the shallow water range (Kaihatu and Kirby 1995). This is rigorous test for dispersive wave models. Figure 2 shows the experimental setup of Mase and Kirby (1992). In this experiment, the free surface elevation has been measured in the sampling rate of $20 \mathrm{~Hz}$. The dataset is divided into 7 realizations and each realization has 2048 data points. The peak frequency of the free surface spectra is $1 \mathrm{~Hz}$ and the total number of frequency components taken for this analysis is 400 . Figures 3 and 4 compares the laboratory measurement with the real amplitude model and the model of Kaihatu and Kirby (1995).

The comparison of models shows that the real amplitude model agrees very well with the data. The difference between this model and the model of Kaihatu and Kirby (1995) shows that the real amplitude model has a significant improvements in resolving higher frequencies particularly at nearshore gages compare to other model.

The second dataset is that of the Bowen and Kirby (1994) which is used to analyze the behavior of the model with the smaller range of relative water depth. Figure 5 shows the experimental setup of Bowen and Kirby (1994).This data set, herein after BK94, provides three different wave conditions. The free surface elevation was measured at 47 gages with the sampling rate of $25 \mathrm{~Hz}$ for the duration of $17 \mathrm{~min}$. The dataset is divided into 12 realizations with 2048 data points in each. Case B of BK 94 is used for this comparison. In this case, the $H_{r m s}$ and the peak frequency, $f_{p}$ are $0.08 \mathrm{~m}$ and $0.225 \mathrm{~Hz}$ respectively. Figure 6 compares real amplitude models; the model of Kaihatu and Kirby (1995) and the model of Freilich and Guza (1984) with the experimental dataset.

The results of the comparisons show that the real amplitude model works reasonably well. Although the model of Freilich and Guza (1984) compares better for the lower frequency (nearshore part of the spectra), the real amplitude model has a better agreement at higher frequencies in more nearshore gage locations compare to other two models.

\section{Comparison of the model with field dataset in terms of spectra}

In addition to compare the models with experimental dataset, the model is verified using field data from the Duck 94 experiment which was conducted at the U.S. Army Field Research Facility at Duck, North Carolina, USA in the fall of 1994 (Birkemeier and Thornton 1994). Figure 7 shows the schematic view of the Duck 94 experimental field. The field experiments capture a wide range of conditions than seen in the laboratory. In this test, we examine in detail the accuracy of the model for different cases of wave 

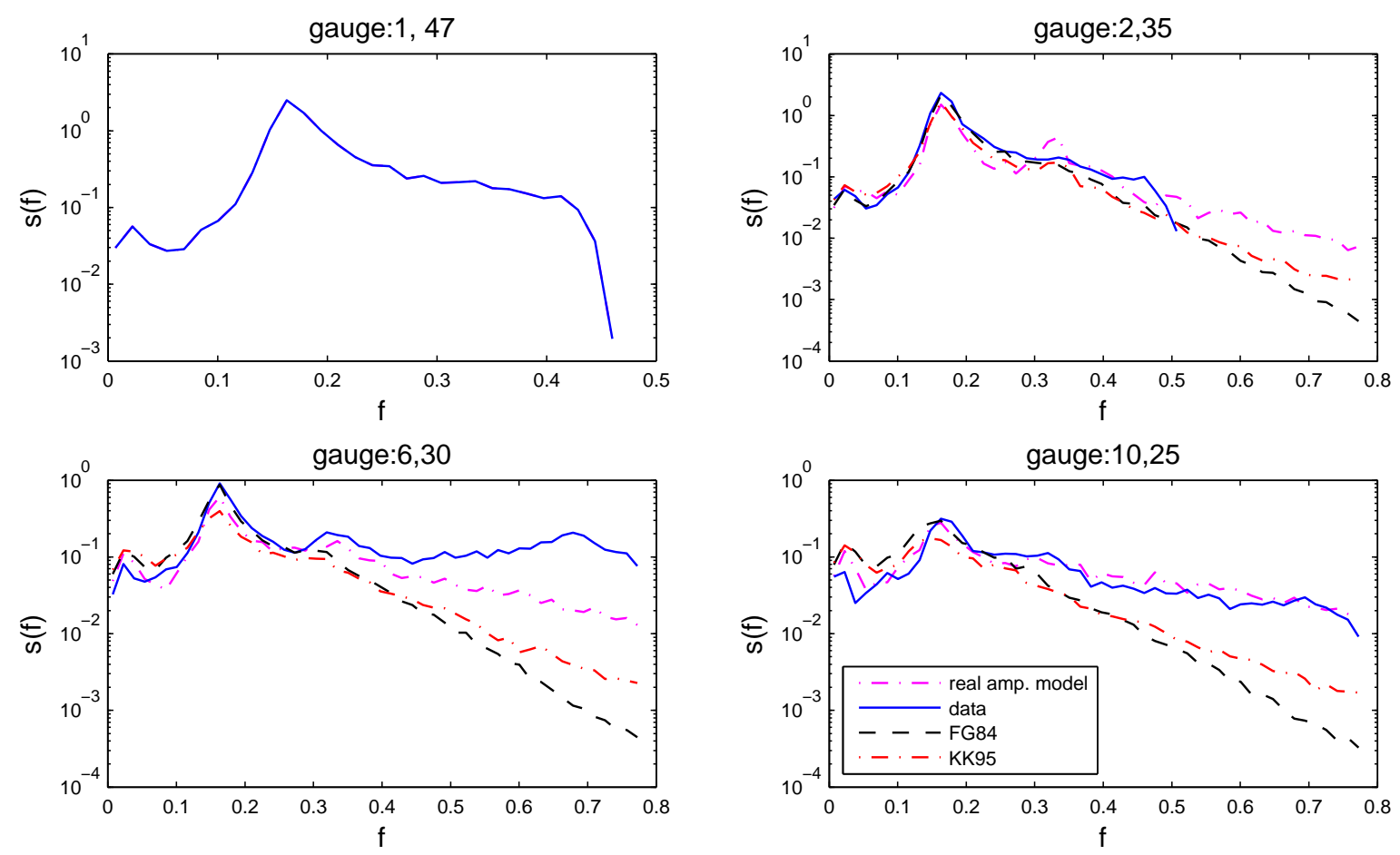

Figure 8: Comparison of Duck94-09030100 field dataset with real amplitude model, Kaihatu and Kirby (1995) mild slope model and the consistent model of Freilich and Guza (1984)-400 frequency components

height and peak frequency. Figure 8 presents the comparison of nearshore wave models with Duck 94 field data for 400 retained frequency components that is carried out for 3 September 1994 at 0100 Hrs EST. It is clearly seen that the real amplitude model for this case, predicts the behavior of the waves better than other models particularly for nearshore gages at higher frequencies.

\section{CONCLUSION}

The aim of the present study was developing a fully dispersive wave model that improves the prediction of high frequency tail of the wave spectra. To do this, we have derived a deterministic model based on the reformulation of the dispersive model of Kaihatu and Kirby (1995). This frequency domain model includes the triad wave-wave interaction terms in the second order. To take into account the dissipation mechanism due to the breaking waves, the dissipation term based on the formulation of Thornton and Guza (1983) was added to the model.

The developed model (also called real amplitude model) has been verified numerically using the comparisons with either experimental or field data. The comparisons of free surface elevation amplitude for the first four harmonics with the experimental data of Chapalain et al. (1992) demonstrate that the real amplitude model has relatively good agreement with observed data.

The comparisons of the model-predicted spectra with laboratory experiments shows that the model accurately predicts the high frequency tail of spectra compare to the model of Kaihatu and Kirby (1995) and the consistent model of Freilich and Guza (1984). The variety of test cases for different wave conditions confirms that the model is verified to predict wave conditions with a wide range of Ursell numbers.

\section{ACKNOWLEDGMENT}

This project was funded by ONR Grant No.N00014-10-1-0389. The authors would like to thank Drs. Steve Elgar, Robert Guza, William O' Reilly and the staff of the U.S. Army Corps Field Research Facility for the data used in this study. 


\section{References}

Y. Agnon and A. Sheremet. Stochastic nonlinear shoaling of directional spectra. Journal of Fluid Mechanics, 345:79-99, 1997.

Y. Agnon, A. Sheremet, J. Gonsalves, and M. Stiassnie. Nonlinear evolution of a unidirectional shoaling wave field. Coastal Engineering, 20(1):29-58, 1993.

S. Ardani. A Model for Transformation of Fully Dispersive Nonlinear Waves. PhD thesis, 2016.

T. B. Benjamin, J. L. Bona, and J. J. Mahony. Model equations for long waves in nonlinear dispersive systems. Phil.Trans.R.Soc.Lond.A, 272(1220):47-78, 1972.

D. Benney. Non-linear gravity wave interactions. Journal of Fluid Mechanics, 14(04):577-584, 1962.

W. A. Birkemeier and E. B. Thornton. The duck94 nearshore field experiment. In Coastal Dynamics $\tilde{A} \hat{a}, \neg 94$, pages $815--821 . A S C E, 1994$.

B. Boczar-Karakiewicz, J. Bona, and D. Cohen. Interaction of shallow-water waves and bottom topography. 1986.

B. Boczar-Karakiewicz, J. Bona, and D. Cohen. Interaction of shallow-water waves and bottom topography. Institute for Mathematics and Its Applications, 4:131, 1987.

N. Booij, R. Ris, and L. H. Holthuijsen. A third-generation wave model for coastal regions: 1. model description and validation. Journal of geophysical research: Oceans, 104(C4):7649-7666, 1999.

J. Boussinesq. Theorie des ondes et des remous qui se propagent le long d'un canal rectangulaire horizontal, en communiquant au liquide contenu dans ce canal des vitesses sensiblement pareilles de la surface au fond. Journal de Mathematiques Pures et Appliques, pages 55-108, 1872.

G. D. Bowen and J. T. Kirby. Shoaling and breaking random waves on a 1:35 laboratory beach. Technical report, 1994.

H. Bredmose, H. Schaffer, and P. A. Madsen. Boussinesq evolution equations: Numerical efficiency, breaking and amplitude dispersion. Coastal Engineering, 51(11):1117-1142, 2004.

P. Bryant. Stability of periodic waves in shallow water. Journal of Fluid Mechanics, 66(01):81-96, 1974.

P. J. Bryant. Periodic waves in shallow water. Journal of fluid mechanics, 59:625-644, 1973.

G. Chapalain, R. Cointe, and A. Temperville. Observed and modeled resonantly interacting progressive water-waves. Coastal Engineering, 16(3):267-300, 21992.

Y. Chen and P. L.-F. Liu. Modified boussinesq equations and associated parabolic models for water wave propagation. Journal of Fluid Mechanics, 288:351-381, 1995.

V. H. Chu and C. C. Mei. On slowly-varying stokes waves. Journal of Fluid Mechanics, 41(04):873-887, 1970.

Y. Eldeberky. Nonlinear effects in gravity waves propagating in shallow water. Coastal Engineering Journal, 54(04), 2012.

Y. Eldeberky and J. A. Battjes. Spectral modeling of wave breaking: Application to boussinesq equations. Journal of Geophysical Research: Oceans, 101(C1):1253-1264, 1996.

Y. Eldeberky and P. A. Madsen. Deterministic and stochastic evolution equations for fully dispersive and weakly nonlinear waves. Coastal Engineering, 38(1):1-24, 1999.

M. H. Freilich and R. T. Guza. Nonlinear effects on shoaling surface gravity waves. Philosophical Transactions of the Royal Society of London (Series) A: Mathematical and Physical Sc, 311(1515):1-41, 1984. 
T. Janssen, T. Herbers, and J. Battjes. Generalized evolution equations for nonlinear surface gravity waves over two-dimensional topography. Journal of Fluid Mechanics, 552:393-418, 2006.

B. Kadomtsev and V. Petviashvili. On the stability of solitary waves in weakly dispersing media. In Sov. Phys. Dokl, volume 15, pages 539-541, 1970.

J. M. Kaihatu. Improvement of parabolic nonlinear dispersive wave model. Journal of waterway, port, coastal, and ocean engineering, 127(2):113-121, 2001.

J. M. Kaihatu. Frequency domain wave models in the nearshore and surf zones. Amsterdam: Elsevier, 2003.

J. M. Kaihatu and J. T. Kirby. Nonlinear transformation of waves in finite water depth. Physics of Fluids, 7(8):1903-1914, 1995.

J. M. Kaihatu and J. T. Kirby. Effects of mode truncation and dissipation on predictions of higher order statistics. Coastal Engineering Proceedings, 1(25), 1996.

J. M. Kaihatu and J. T. Kirby. Two-dimensional parabolic modeling of extended boussinesq equations. Journal of waterway, port, coastal, and ocean engineering, 124(2):57-67, 1998.

J. M. Kaihatu, J. Veeramony, K. L. Edwards, and J. T. Kirby. Asymptotic behavior of frequency and wave number spectra of nearshore shoaling and breaking waves. Journal of Geophysical Research: Oceans

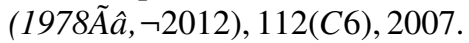

J. M. Kaihatu, S. Ardani, J. T. Goertz, A. Venkattaramanan, and A. Sheremet. Wave dissipation mechanisms in spectral phase-resolved nonlinear wave models. Advances in Coastal Hydraulics, page 235, 2018.

J. B. Keller. Resonantly interacting water waves. Journal of Fluid Mechanics, 191:529-534, 1988.

J. T. Kirby and J. M. Kaihatu. Structure of frequency domain models for random wave breaking, 1996.

J. T. Kirby, J. M. Kaihatu, and H. Mase. Shoaling and breaking of random wave trains: spectral approaches. In Engineering Mechanics, pages 71-74. ASCE, 1992.

D. J. Korteweg and G. D. Vries. Xli. on the change of form of long waves advancing in a rectangular canal, and on a new type of long stationary waves. The London, Edinburgh, and Dublin Philosophical Magazine and Journal of Science, 39(240):422-443, 1895.

P. L. F. Liu and M. W. Dingemans. Derivation of the third order evolution equations for weakly nonlinear water waves propagating over uneven bottoms. Wave motion, 11:41-64, 1989.

P. L.-F. Liu and T.-K. Tsay. Refraction-diffraction model for weakly nonlinear water waves. Journal of Fluid Mechanics, 141:265-274, 1984.

P. L.-F. Liu, S. B. Yoon, and J. T. Kirby. Nonlinear refraction-diffraction of waves in shallow water. Journal of Fluid Mechanics, 153:185-201, 1985.

P. A. Madsen and Y. Eldeberky. A new formulation of deterministic and stochastic evolution equations for three-wave interactions involving fully dispersive waves. Coastal Engineering Proceedings, 1(26), 1998.

P. A. Madsen and O. R. Sorensen. A new form of the boussinesq equations with improved linear dispersion characteristics. part 2. a slowly-varying bathymetry. Coastal Engineering, 18(3):183-204, 1992.

P. A. Madsen, R. Murray, and O. R. Sorensen. A new form of the boussinesq equations with improved linear dispersion characteristics. Coastal Engineering, 15(4):371-388, 1991.

H. Mase and J. T. Kirby. Hybrid frequency-domain kdv equation for random wave transformation. Coastal Engineering Proceedings, 1(23), 1992.

C. Mei and B. L. Mehaute. Note on the equations of long waves over an uneven bottom. Journal of Geophysical Research, 71(2):393-400, 1966. 
C. C. Mei and U. Unluata. Harmonic generation in shallow water waves. Waves on Beaches and Resulting Sediment Transport, pages 181-202, 1972.

O. Nwogu. Alternative form of boussinesq equations for nearshore wave propagation. Journal of waterway, port, coastal, and ocean engineering, 119(6):618-638, 1993.

D. Peregrine. Long waves on a beach. Journal of Fluid Mechanics, 27(04):815-827, 1967.

O. Phillips. The dynamics of the upper ocean cambridge university press. New York, 1977.

A. Radder. On the parabolic equation method for water-wave propagation. Journal of Fluid Mechanics, 95 (1):159-176, 1979.

J. Roelvink and M. Stive. Bar-generating cross-shore flow mechanisms on a beach. Journal of Geophysical

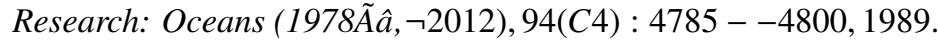

R. Smith and T. Sprinks. Scattering of surface waves by a conical island. Journal of Fluid Mechanics, 72 (02):373-384, 1975.

K. D. Suh, R. A. Dalrymple, and J. T. Kirby. An angular spectrum model for propagation of stokes waves. Journal of Fluid Mechanics, 221:205-232, 1990.

E. B. Thornton and R. Guza. Transformation of wave height distribution. Journal of Geophysical Research: Oceans (1978-2012), 88(C10):5925-5938, 1983.

H. L. Tolman, M. L. Banner, and J. M. Kaihatu. The nopp operational wave model improvement project. Ocean Modelling, 70(0):2-10, 102013. 